\title{
Application of BP Neural Networks to Weigh-in-Motion of Vehicles
}

\author{
Zhifeng Zhou \\ School of Mechanical Engineering \\ Shanghai University of Engineering Science \\ No.333 Longteng Road, 201620 Shanghai, China \\ zhousjtu@126.com
}

\begin{abstract}
BP neural networks are employed to estimate the static axle weight of moving vehicles. On the basis of analyzing the characteristics of dynamic tire force, the influence of dynamic tire force is briefly introduced on the weighing accuracy. The three-layer BP neural networks are designed to process the axle weight signal. The selection of parameters of neural networks is analyzed. The weighing data of three two-axle trucks are used to train and test the developed neural networks. The results show that the proposed three-layer BP neural networks are effective. The max axle weight error is less than $5.18 \%$.
\end{abstract}

Keywords- Weigh-In-Motion; BP neural networks; Dynamic tire force

\section{INTRODUCTION}

With the rapid development of traffic and transportation, the increasing overweight vehicles have become a serious problem, which shorten the lifespan of roads and bridges, cause traffic accidents and result in great loss in the state's tax income. Jinfang Wang ${ }^{[1]}$ introduces the various harms of the overweight vehicles. In order to improve safety on road, protect road infrastructure and enforce traffic law, it is necessary and important to develop Weigh-In-Motion (WIM) method to measure axle weight of vehicles reliably, accurately and fast.

Many factors affect the measurement accuracy of the axle weight. F.Scheuter ${ }^{[2]}$ discusses the influences of vehicle suspension, vehicle speed, tyre spring and road evenness on the measuring accuracy. Various approaches have been proposed to improve weighing accuracy. M.Niedzwiecki ${ }^{[3]}$ compares the performances of simple maximum value detection method, system identification method and extended Kalman filtering method on laboratory models. Mangeas. $\mathrm{M}^{[4]}$ uses feed forward neural networks to fuse multiple-sensor measurements into a single value and improves estimation accuracy of the static weight. D.Cebon ${ }^{[5]}$ introduces the Multiple-Sensor Weigh-in-motion (MS-WIM) system in which many strip sensors are installed along a length of 25 to $50 \mathrm{~m}$ in the road. These two methods are based on the fusion of multiple-sensor measurements. The design of sensor array and the calibration of sensors are difficult.

Artificial neural networks possess strong nonlinear mapping, adaptive learning, fault-tolerance performance and robustness. They are widely used in pattern recognition, data processing, fault diagnosis, and so on. In the following sections, the characteristics of dynamic tire forces are analysed; the three-layer BP neural networks are employed to process WIM data; the pre-processing of the signals of WIM is performed; and the selection of parameters is analysed in neural networks. Real experiments are conducted to evaluate the performance of the proposed BP neural networks.

\section{DYNAMIC TIRE FORCES}

When vehicle keeps static, the force of tires exerting on the ground is equal to the static axle weight (which is regarded as the real axle weight in practice). When vehicle moves, the tire force exerting on the ground contains the dynamic tire forces beside the static axle weight. The quarter-car model ${ }^{[6]}$ is often used to study the dynamic tire forces. Fig.1 shows the quarter-car model.

Referencing the approach described in Appendix B of [6], we can analyze the characteristics of dynamic tire forces by data simulation. Zhou zhifeng ${ }^{[7]}$ uses quarter-car model and road roughness model to simulate dynamic tire forces. The dynamic tire forces are a kind of low frequency signals whose amplitude can reach $30 \%$ of the real axle weight and lowest frequency can reach $1.5 \mathrm{~Hz}$. The amplitudes and frequencies of dynamic tire forces vary with vehicle speed, load of vehicle, the position of load, vehicle suspension, tyre spring and road evenness, and so on. When the width (direction of vehicle movement) of scale platform is certain, the quicker the vehicle speed the shorter the sampling time. For example, when the width of weighing platform is $760 \mathrm{~mm}$, the available width is about $490 \mathrm{~mm}$ taking account of the tire-pavement contact area. When the vehicle runs across the platform at $10 \mathrm{~km} / \mathrm{h}$, the valid sampling time is $178 \mathrm{~ms}$ and only 0.54 times of the cycle of the $3 \mathrm{~Hz}$ dynamic tire force. The whole cycles of the dynamic forces can not be obtained during the finite sampling time.

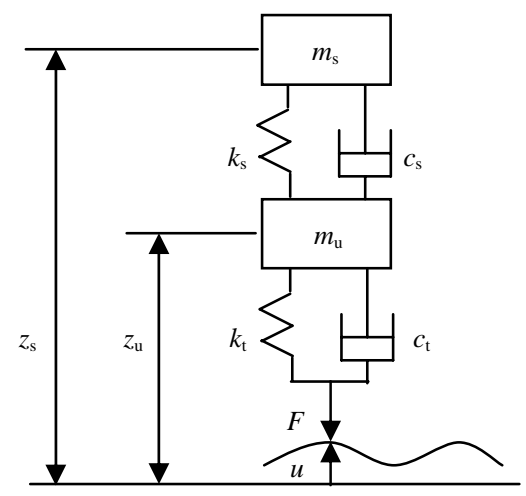

Fig.1 Quarter-car model

$m_{\mathrm{s}}:$ Sprung mass 
$m_{\mathrm{u}}$ : Unsprung mass

$c_{\mathrm{s}}$ : Suspension damping

$c_{\mathrm{t}}$ : Tire damping

$k_{\mathrm{s}}$ : Suspension stiffness

$k_{\mathrm{t}}$ : Tire stiffness

$Z_{s}$ : Displacement of ms from the static equilibrium position

$z_{\mathrm{u}}$ : Displacement of $\mathrm{mu}$ from the static equilibrium position

$u$ : Displacement of road profile input

$F$ : Tire force exerting on the ground

\section{STRUCTURE OF BP NEURAL NETWORKS}

It has been theoretically proved that three-layer neural networks can realize arbitrarily complicated nonlinear mapping problems [6]. BP neural networks are a kind of multilayer forward neural networks based on back propagation algorithm. It is reported that about 90\% applications of artificial neural networks are based on BP algorithm. In this paper, we develop a three-layer BP neural network shown in Fig.2 to process the axle weight signal of vehicle in motion. In Fig.2, $x_{i}(1 \leq i \leq 4)$ denotes the element of input vector, $b_{i}(1 \leq i \leq 10)$ denotes the bias values of neurons in hidden layer, $b$ denotes the bias value of the output neuron, $w_{m j}(1 \leq m \leq 4,1 \leq j \leq 10)$ denotes the synaptic weights of neurons between the input layer and hidden layer, $w_{j 1}(1 \leq j \leq 10)$ denotes the synaptic weights of neurons between the hidden layer and output layer, $w$ denotes the estimation of static axle weight.

The inputs of neural networks should contain the information of the weighed vehicle if possible. One approach is to directly regard the axle weight signals as the input vectors of neural networks. When the sampling interval is certain, the dimensions of input vectors will be not equal since the real velocities of vehicle are impossibly equal every time, which will result in the failure of neural networks. Furthermore, too many inputs of the input layer will influence the real-time performance of the neural networks. In this paper, the velocity, the wheelbase, the average value and the maximum value of the axle weight signal are selected as the inputs.

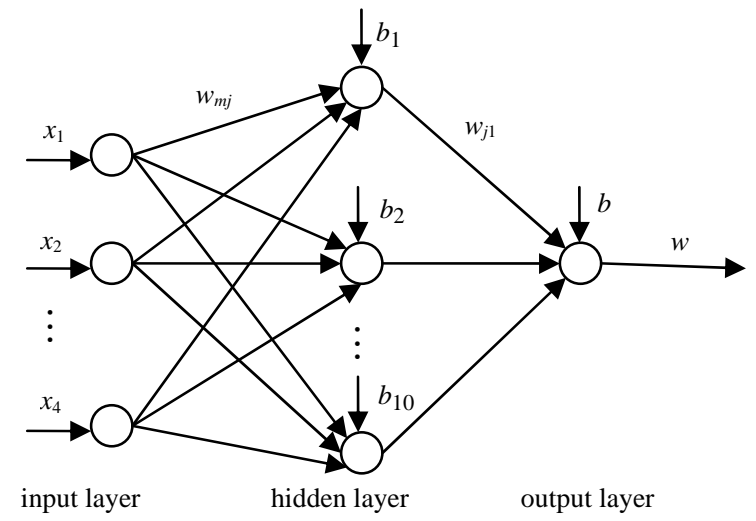

Fig.2 Structure of the three-layer BP neural networks for WIM
It is difficult to confirm the number of neurons in hidden layer. The accuracy can be improved by increasing the number of neurons in hidden layer, but it will bring vast calculation with the increasing of number of neurons, which willinfluence the real-time application of neural networks. According to the empirical formula and the experiment research, the number of neurons in hidden layer is 10 in this paper.

The transfer function of neurons in hidden layer can be described by

$$
f_{1}(x)=\frac{2}{1+\exp (-2 x)}-1
$$

The outputs of neurons in hidden layer can be written as

$$
M=f_{1}\left(X W_{1}+B\right),
$$

where $M=\left[m_{1}, m_{2}, \cdots, m_{10}\right], X=\left[x_{1}, x_{2}, x_{3}, x_{4}\right]$,

$$
W_{1}=\left[\begin{array}{ccc}
w_{1,1} & \cdots & w_{1,10} \\
\vdots & \vdots & \vdots \\
w_{4,1} & \cdots & w_{4,10}
\end{array}\right], B=\left[b_{1}, b_{2}, \cdots, b_{10}\right] .
$$

The transfer function of output layer can be presented as

$$
f_{2}(x)=x \text {. }
$$

The estimation of the static axle weight can be written as

$$
w=f_{2}\left(M W_{2}+b\right),
$$

where $W_{2}=\left[w_{1,1}, w_{2,1}, \cdots, w_{10,1}\right]$.

\section{PREPROCESSING OF THE SAMPLED SigNALS}

In real experiments, the scale platform has dimensions of $3 \mathrm{~m}$ and $0.75 \mathrm{~m}$ for length and width (direction of vehicle movement). Three two-axle trucks (named A, B and C) are used. Table I lists the times of trucks crossing the scale platform at the different speeds with the different loads. The sampling frequency is $10 \mathrm{KHz}$.

Taking account of the contact area of the tyre, we think the first maximum point of the ascent segment denote tyre's entering the scale platform entirely and the first maximum point of the descent segment denote tyre's beginning to leave the platform. We select the data segment between the two maximum points as the axle weight signal, and computing the average value and the maximum value of the axle weight signal. The dashed line depicts the selection of the axle weight signal in Fig.3.

TABLE I times of trucks crossing the scale platform at different speeds with different loads

\begin{tabular}{|c|c|c|c|c|c|c|}
\hline \multicolumn{2}{|c|}{} & \multicolumn{5}{|c|}{ Velocity (km/h) } \\
\cline { 3 - 7 } \multicolumn{2}{|c|}{} & 10 & 15 & 20 & 25 & 30 \\
\hline Truck A (times) & $1000 \mathrm{~kg}$ load & 40 & 40 & 40 & 40 & 40 \\
\hline \multirow{3}{*}{ Truck B (times) } & $1000 \mathrm{~kg}$ load & 29 & & 29 & & 29 \\
\cline { 2 - 7 } & $2000 \mathrm{~kg}$ load & 29 & & 29 & & 29 \\
\hline \multirow{3}{*}{ Truck C (times) } & $0 \mathrm{~kg}$ & 23 & & 23 & & 23 \\
\cline { 2 - 7 } & $15000 \mathrm{~kg}$ load & 23 & & 23 & & 23 \\
\hline
\end{tabular}




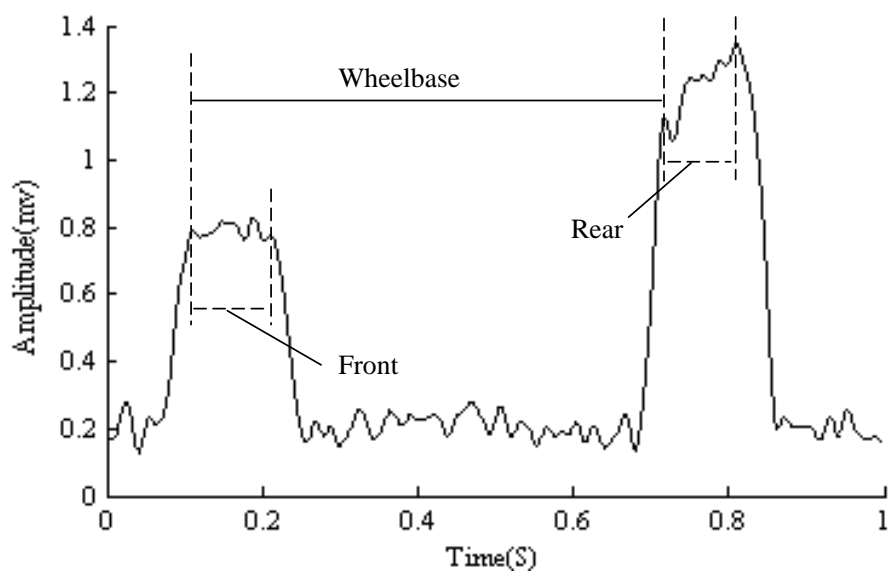

Fig.3 Selection of the axle weight signal from the sampled signal

Since we can't know the velocity and wheelbase of the trucks crossing the scale platform in real practice, we compute the velocity and wheelbase using the sampled signal. According to the sampling interval, the length of the axle weight signal, the width of the scale platform and the interval of front axle and rear axle crossing the scale platform, we can calculate the vehicle velocity and the wheelbase. The sample vector of the BP neural networks consists of the average value, the maximum value, the velocity and the wheelbase. We can extract two sample vectors from every sampled signal. After preprocessing, we obtain 400 sample vectors of truck A, 348 sample vectors of truck B and 276 sample vectors of truck C.

\section{REAL SIGNALS EXPERIMENTS}

In order to testing the performance of the developed BP neural networks, two kinds of experiments are performed. Three trucks' sample vectors are trained and tested, respectively. The $90 \%$ sample vectors of truck A are randomly selected as the training samples and the other $10 \%$ sample vectors are employed as the test samples. The $90 \%$ sample vectors of truck $B$ are randomly selected as the training samples and the other $10 \%$ sample vectors are employed as the test samples. The $90 \%$ sample vectors of truck $\mathrm{C}$ are randomly selected as the training samples and the other $10 \%$ sample vectors are employed as the test samples. The max axle weight errors of the test samples of truck A, truck B and truck $\mathrm{C}$ are less than $0.61 \%, 1.45 \%$ and $0.85 \%$, respectively.

The total sample vectors of three trucks are split into two parts. One part (921 samples are extracted from the total sample vectors, in which 360 samples are randomly extracted from truck A, 313 samples are randomly extracted from truck B and 248 samples are randomly extracted from truck C) is used to train BP neural networks. The other part (103 remainder samples) is used to test the performance of the trained BP neural networks. The max axle weight errors of the test samples of truck $\mathrm{A}$, truck $\mathrm{B}$ and truck $\mathrm{C}$ are less than $3.48 \%$, $5.18 \%$ and $2.46 \%$ respectively.

It can be see that the developed BP neural networks are effective. When the training samples and the test samples come from the same truck, the max error is $1.45 \%$; when the training samples come from three trucks and the test samples come from the remainder samples of three trucks, the max error is $5.18 \%$.

\section{CONCLUSION}

In this paper, the factors affecting the weighing accuracy of the axle weight are briefly introduced. The three-layer BP neural networks containing four input neurons, ten hidden layer neurons, and one output neuron, are developed. The average value and max value of the axle weight signal, the velocity and the wheelbase are regarded as the inputs of $\mathrm{BP}$ neural networks. By preprocessing, the samples of three two-axle trucks are used to train and test the neural networks. The results show that the developed BP neural networks are effective, and the max axle weight error is less than $5.18 \%$.

\section{ACKNOWLEDGMENT}

The work described in this paper was supported by 085 knowledge innovation project of Shanghai University of Engineering Science (Project No. JZ0906).

\section{REFERENCES}

[1] Jinfang Wang, Mingguang Wu, "An overview of research on weigh-in-motion system", Proceeding of the $5^{\text {th }}$ Word Congress on Intelligent Control and Automation, 2004, pp. 5241-5244.

[2] F. Scheuter, "Evaluation of factors affecting WIM system accuracy," Proc. of the 2nd European Conf. on WIM, Lisbon, 1998, P1216, E0-E9

[3] M.Niedzwiecki, A.Wasilewski, "Application of Adaptive Filtering to Dynamic Weighing of Vehicles”, Control Eng. Practice, vol. 4, no. 5, pp. 635-644, 1996.

[4] Mangeas.M, Glaser.S and Dolcemascolo.V, "Neural networks estimationof truck static weights by fusing weight-in-motion data", Proceedings of the Fifth International Conference on Information Fusion, 2002, pp. 456-462.

[5] D. Cebon, "WAVE Report of WP1.1: Multiple Sensor WIM," 2001, pp.1-73 (http://wim.zag.si/wave/download/wp11_final.pdf, Last accessed Mar. 13, 2010).

[6] D. Cebon, "Interaction between Heavy Vehicles and Roads," SAE International, 1993, pp.1-82.

[7] Zhou zhifeng, Hu xiujuan, "Characteristics of Dynamic Tire Force in Weigh-in-Motion”, Journal of Shanghai University of Engineering Science, vol.23, no.1, pp.32-36. 